\title{
Ontology as a Natural Extension of Predicate Calculus with Identity Equipped with Description
}

\author{
Toshiharu WaragaI
}

\section{Introductory}

The main aim of this paper is to show that a natural extension of the predicate calculus with identity (hereafter LI) enriched with some logico-linguistically proper devices leads us in quite a natural way to the axiom of Leśniewski's Ontology, and that Ontology in turn implies the theses of the LI thus enriched. In addition the degrees of extensionality of the enriched LI will be discussed.

In showing this, the Russellian notion of description appropriately modified will play an essential role. Indeed it is evident that, in its semantic intention, the axiom of Ontology is quite similar to the Russellian theory of description. ${ }^{1} \mathrm{Hiz}$ [1977] shows in fact that the Russellian definition of description is inferentially equivalent to the axiom of Ontology in a sense to be specified ${ }^{2}$, namely for semantic categories higher than that of names.

The present paper will show the logical fact that LI suitably enriched is inferentially equivalent to Ontology for the lowest semantic category as well. As a consequence Leśniewski's Ontology is not and should not be regarded as a system which is rather unique and specific, being isolated from the usually accepted logical framework. It is a logically natural extension of Frege-Russellian logical system with identity and description. It is something which could well have been proven before, but as far as I know it has not yet been proven. This is certainly because of the ontological prejudice which has dominated the main stream of logical analysis since the very time when it was created by its founders ${ }^{3}$.

\section{Some Defects of Predicate Calculus with Identity}

LI is evidently defective in a couple of points, even when it is considered as a tool for describing extensional logical aspects of natural language.

1.1. The first point is related to the notion of names. What LI allows as names are

Tokyo Institute of Technology, Department of Philosophy and Logic, O-okayama Meguroku Tokyo 152 Japan

1. On similarity and dissimilarity, cf. Lejewski [1960].

2. Cf. Hiz [1977], p. 272.

3. Cf. Waragai [1987]. 
only individual names, and LI lacks the ability to deal with names in general in a proper way. For example the general name 'man' finds no proper place in LI in its own right. It must be converted to the verb 'is-a-man' to be treated within the framework of LI. Thus it never appears as a separate, autonomous unit. Names in LI constitute so to speak purely syncategorematic ${ }^{4}$ part of LI, getting their syntactic role only as a mere constitutive part of verbs. ${ }^{5}$. And that is without doubt an inconvenient and clumsy feature of LI which should be overcome in some appropriate way if we want logic to be closer to natural language.

Thus let us accept a new ${ }^{6}$ position concerning names in order to get rid of this inconvenience. Namely, we widen the semantic category of names to general names to include 1) general designative names, 2) singular names and 3) empty names. In addition a name can be structurally a) simple or b) complex ${ }^{7}$. A definite description is a name which is $2 b$ ), i.e. singular and complex. Widened in this way, names get proper autonomous status in LI.

1.2. A point which arises due to this conceptual change in names is the logical status of the copula 'is'. Three or four different usages of 'is' are acknowledged in the philosophy of language which accompanies $\mathrm{LI}^{8}$. That is, 1) the 'is' in predicative use, 2) the 'is' in the role of identity, 3) the 'is' in the role of existence, and eventually 4) the 'is' in the role of inclusion'. According to the standard view, they are totally different from each other, and need to be expressed by different symbolism. This implies that one usage is not definable by the other usages. But a close look will show that such a conception apparently runs against our general understanding of the logical grammatical structure and function of singular propositions of the form ' $A$ is $b$ ', where 'is' appears in predicative use.

The singular-predicative use of 'is' seems to be logico-semantically prior to the other ways of its use, because the 'is' in the role of identity, the 'is' in the role of

4. I call an expression purely syncategorematic if it has no semantic category assigned. This differes from the standard usage of 'syncategorematic'. On the standard usage of the term, cf. e.g. Gochet [1980], p. 15.

5. Usually with the exception of singular simple terms, i.e. proper names or individual constants. But notice that this theoretical attitude reached its extreme in Quine [1948]. He shunned there even proper names, converting them to descriptive terms.

6. It is in fact not new by any means. Our natural intuition of language has been well aware of this fact, and to those who accept the Leśniewskian idea of language, this has been from the beginning the most natural attitude toward names. The point is that this unnatural view to restrict the semantic category of names only to that of singular ones has gained rather uncritical support for a relatively long time and the traditional view has been almost totally forgotten since the birth of LI.

7. Cf. e.g. Lejewski [1958]. Cf. also Kaplan [1970], esp. pp. 284-6 where he discusses what it is for a semantics of a language to be perfect.

8. Cf. Hintikka [1983].

9. Notice that the 'is' in the role of inclusion gets a natural sense when we have at hand general names or sets. 
existence as well as the 'is' in the role of inclusion are definable in terms of the singular-predicative 'is'. Let us write the singular-predicative 'is' as ' $i s^{\prime}$ '. Now we say ' $a$ is $b$ ' when and only when $a$ is an object such that it is in fact $b$. This is the truth condition under which the 'is' in the predicative role is used.

Now the other three ways of use of 'is' can be defined in the following way: 1) we say that $a$ is-identical-with $b$ when and only when $a$ is $b$ and $b$ is $a$; 2) we say that there is such a thing as $F$ when and only when there is some object such that it is $F$. 3) We say that $a$ is included in $b$ when and only when for every object $x$ if $x$ is $a$ then $x$ is $b$. What is more, a close examination of the truth condition of singular-predicative propositions tells us that we say that $a$ is an object when and only when $a$ is $a$. Thus, once we have the predicative 'is' at hand, the others are definable, so that there seems no alleged discrepancy between the different usages of 'is'.

Following that intuition, we admit an autonomous status for the predicative 'is' in propositions of the form ' $A$ is $b^{\prime 11}$. For this reason, we introduce into LI the functor ' $\varepsilon$ ' of the semantic category $s / n, n$, i.e. the proposition-forming functor of two name which is intended to be the formal correspondent of 'is' in the role of predicative use. Thus a proposition of the form ' $A \varepsilon b$ ' is designed to be a singular proposition which states of $A$ that it is an object such that it is $b$. Since singular propositions play an essential role in saying of an object that it is such-and-such, I take this functor as fundamental in this paper.

1.3. Accepting a logically autonomous status for the copula 'is' in predicative use, we have to admit at the same time another operation which is familiar to natural language. That is the conversion of a name to the verb corresponding to it. For example, we get the verb 'is-a-man' from the name 'man'. Thus we must have an appropriate device for this operation. For this purpose let us introduce a functor of the semantic category $(s / n) / n$. And let us express that by ' $\varepsilon$ ' in equiform to the above introduced functor ' $\varepsilon^{\prime 12,13}$. This functor ' $\varepsilon$ ' takes as its argument a name, and

10. Notice that this ' $i s$ ' in used in a technical way, and there is no exact correspondent in colloquial English. In Japanese there is no correspondent. This ' $i$ ' ' is to be regarded as a logical reconstruct of everyday singular proposition formative ' $i s$ '.

11. I find the comment in Lejewski [1960] on what the theory of description is about of great interest. He suggests there that the theory of description is rather concerned with the analysis of 'is' than the analysis of the definite article 'the'. See p. 16.

12. There are some other copulas of the category $(\mathrm{s} / \mathrm{n}) / \mathrm{n}$. Cf. for example Lejewski [1956]. Many copulas are there dealt with. The copulas dealt with there are of the category $s / n, n$. But their analogues of the category $(s / n) / n$ are easy to introduce.

13. Strictly speaking, this functor should be expressed by another symbol, e.g. ' $\varepsilon_{1}$ ', since then ' $\varepsilon$ ' would belong to two different semantic categories at the same time. But as the number of semantic categories that appear in this paper is limited, the ambiguous use should not cause any problem. 
forms a verb corresponding to the name. I stipulate that ' $\varepsilon+a f^{\prime}$ ' is to be read ' $i s-a$ ', a verb corresponding to the name ' $a$.'

1.4. Another shortcoming of LI is that it lacks the ability to construct a name from a verb. Natural language is equipped with devices by means of which a verb is converted to a name corresponding to it. For example, from the verb 'is-a-man' we construct the name 'that which is-a-man'14. But LI is not equipped with such a device. This is also without doubt a shortcoming which it is desirable to remedy by some logical device.

For this purpose, let us introduce the functor 'trm' of the category $n /(s / n)$, a functor producing a name taking a verb as its argument. A verbal rendering of 'trm' is 'that which', so that it converts a verb ' $\varepsilon+a f$ ' to the name 'trm $\langle\varepsilon+a f\rangle$ '. In general, a verb or a predicate ' $\phi$ ' is converted to the name 'that which $\phi$ '. Thus ' $\phi$ ' is converted to the name ' $\operatorname{trm}\langle\phi\rangle$ '.

1.5. The last shortcoming of LI on which I want to put special stress in the present paper is concerned with the semantics usually attached to LI.

The logical behavior of the identity in LI is determined by the following axioms :

AI0 $[a](a=a)$

AI1 $[a b](a=b . \supset . b=a)$

$\mathrm{AI} 2[a b c](a=b . b=c . \supset . a=c)$

AI3 $[\phi a b]\left(a=b . \supset .(\phi(a) . \equiv . \phi(b))^{15}\right.$

The identity appearing in LI is usually connected with existence. And it is usual to take ' $[E x](x=a)$ ' or its LI-equivalent ' $a=a$ ' as stating 'there is an object $a^{\prime}$ or ' $a$ is an object'16.

But recall now that we have widened the semantic category of names to include names in the widest sense. Now a careful examination of the axioms will show soon that the identity that is syntactically regulated by these axioms need not necessarily be connected with the notion of existence. That is evidently a matter that belongs to semantics. It is held to be so only because of the view usually accepted concern-

14. This is a name that is general and complex. Another example of such a device is participle. E.g. in Latin the name 'currens (runner, that which runs)' is constructed from 'currere (to run)'. For more on this point, cf. e.g. Henry [1972].

15. AI2 and AI3 are naturally deducible from the others.

16. There is a good philosophical reason to hold that ' $a$ is-identical-with itself' is equivalent to ' $a$ is an individual object'. An argument for it is in Waragai [1985]. The reason I put forward for it is very different from that which is usually given on the basis of Quinian referential interpretation of quantifiers. As for a viewpoint concerning the difference in the manner of ontological commitment between ' $a=a$ ' and ' $[E x](x=a)$ ', cf., e.g. Hintikka [1969]. His viewpoint is supported by the acceptance of referential reading of quantifiers. 
ing the so-called 'existential quantifier' ${ }^{17}$. Indeed AI0, AI1, AI2 are all satisfied by taking ' =' as extensional identity between names, ${ }^{18}$ and as for AI3, if we take an extreme extensionalism in doing logic, it is also satisfied under the same interpretation. Thus seen, the identity in LI is neutral as to ontological commitment if we are ready to reject the dogma of referential interpretation of the quantifier. And the Quinian interpretation of the quantifier as the only vehicle to express the ontological commitment that a theory has seems to be a mere dogma. Apparently the quantifier is only one of the candidates to express existence. Indeed we may well think of some other device to convey the ontological commitment of the theory concerned. And with this change in names, Quinian interpretation breaks down clearly and necessarily. We need another device to state our ontological commitment.

To state the situation in other words, LI fails to distinguish two different kinds of identity. Namely extensional identity and individual identity. The former is free of ontological commitment, while the latter conveys ontological commitment. We have to be clear in this point. Let us keep the identity sign ' $=$ ' of LI for extensional identity. Since it will be shown in the due course that the notion of extensional identity can be defined on the basis of individual identity, let us now pass on to analyzing the notion of individual identity.

\section{An Analysis of Individual Identity}

Let us consider a special case of the 'is' in predicative use 1) the truthcondition of which is :

TC1 ' $a$ is $b$ ' is true iff $a$ is an object such that it is $b^{19}$ and 2) the logical behavior of which is regulated by the following axiom :

AI $[a b](a$ is $b . \equiv .[E x](x$ is $a)$.

$[x y](x$ is b. $y$ is b. $\supset . x$ is $y)$.

$[x](x$ is $a . \supset . x$ is $b)$ )

Under TC1 the following holds:

TC2 If $a$ is $b$ then $a$ is an object.

Thus if we accept the truth condition TC1 concerning the copula ' $i s$ ', the role of ontological burden is conveyed by the formulae of the form ' $a$ is $b$ '.

From this we get the following:

17. In other words, 'particular quantifier with Quinian referential/object-oriented interpretation'.

18. Two names ' $a$ ' and ' $b$ ' are extensionally identical when they have the same extension.

19. Be careful that the 'is' on the left hand appears in technical sense, while the 'is' on the right hand is used in everyday use. 
TC3 If ' $. . a .$. ' implies ' $a$ is $b$ ', then the quantifier in ' $[E x] \ldots x .$. ' may be read in referential way, and we are allowed to read it as 'there is an object such that... it...' Otherwise quantifiers are read simply 'for some' without ontological commitment.

Now as was shown in Waragai [1987], this ' $i s$ ' plays the role of the ' $i s$ ' in the role of individual identity. Thus we are allowed to use AI as an axiom which determines the logical behavior of the 'is' in the role of identity. We will use ' $=$ ' hereafter to express individual identity.

We pose the following as the axiom of individual identity.

A1 $[a b]\left(a={ }_{0} b . \equiv .[E x]\left(x={ }_{0} a\right)\right.$.

$$
\begin{aligned}
& {[x y]\left(x={ }_{0} b \cdot y={ }_{0} b \cdot \supset \cdot x={ }_{0} y\right) .} \\
& \left.[x]\left(x={ }_{0} a \cdot \supset \cdot x={ }_{0} b\right)\right)
\end{aligned}
$$

This can be shortened to:

$\mathrm{A} 1 \#[a b]\left(a={ }_{0} b . \equiv .[E x]\left(x={ }_{0} a \cdot x={ }_{0} b\right)\right)$

which is the axiom Lejewski makes use of in his [1967].

I enumerate some theses concerning individual identity ${ }^{20}$ :

TI1 $[a b]\left(a={ }_{0} b \cdot \supset \cdot b={ }_{0} a\right)$

TI2 $[a b c]\left(a={ }_{0} b \cdot b={ }_{0} c \cdot \supset \cdot a={ }_{0} c\right)$

TI3 $[a b]\left(a={ }_{0} b . \supset . a={ }_{0} a\right)$

TI4 $[a]\left([E x]\left(x={ }_{0} a\right) . \equiv . a={ }_{0} a\right)$

It should be mentioned that A1 is equivalent to the conjunction of TI1 and TI2, with the result that $a={ }_{0} a$ does not hold universally, i.e. $[a]\left(a={ }_{0} a\right)$ is not deducible from $\mathrm{Al}^{21}$.

For this reason, I will refer to individual identity as non-reflexive identity at times $^{22}$.

Thus non-reflexive identity is essentially weaker than the identity usually held in LI. Formally it is identity with total reflexivity deleted. It is this type of identity that we will be concerned with hereafter.

Further we require the axiom of extensionality for individual identity :

A2 $[x y]\left(x={ }_{0} y \cdot \supset \cdot[\phi](\phi(x) . \equiv . \phi(y))\right)^{23}$

The reverse of this thesis holds under some restriction.

20. For proofs of. Waragai [1987].

21. For a proof ef. Waragai [1987].

22. Cf. Lejewski[1967].

23. This is a provable thesis in Ontology without using the axiom of extensionality. Cf. e.g. Shupecki [1955]. 


\section{LI Naturaly Extended}

Let us be concerned with a system which is equipped with non-reflexive identity ' = ${ }_{0}$ ' and two types of ' $\varepsilon$ ' and 'trm' with the syntax of LI appropriately adjusted ${ }^{24}$. Their logical properties will be described below. Let us call this system $\mathrm{LI}^{+}$.

As the axioms regulating ' $={ }_{0}^{\prime}$, we accept $\mathrm{A} 1$ and $\mathrm{A} 2$.

A1 $[a b]\left(a={ }_{0} b . \equiv .[E x]\left(x={ }_{0} a\right)\right.$.

$$
\begin{aligned}
& {[x y]\left(x={ }_{0} b \cdot y={ }_{0} b \cdot \supset \cdot x={ }_{0} y\right) .} \\
& \left.[x]\left(x={ }_{0} a \cdot \supset \cdot x={ }_{0} b\right)\right)
\end{aligned}
$$

A2 $[x y]\left(x={ }_{0} y \cdot \supset \cdot[\phi](\phi(x) . \equiv . \phi(y))\right)$

Now we have to state the logical properties which regulate the newly introduced functors concerning name-verb/verb-name conversion.

Clearly, we have to require that ' $a$ ' and ' $\operatorname{trm}\langle\varepsilon(a)\rangle$ ' be coextensional :

A3 $[\phi a](\phi(a) . \equiv . \phi(\operatorname{trm}\langle\varepsilon f a \jmath\rangle))$

The relation between proposition-forming ' $\varepsilon$ ' and predicate-forming ' $\varepsilon$ ' is the following :

$\mathrm{A} 4 \quad[x a](x \varepsilon a . \equiv . \varepsilon f a f(x))$

That is : $x$ is $a$ if and only if $x$ is-a.

As the basic relation between 'is' in the predicative role and 'is' in the role of individual identity we pose the following:

A5 $[a]\left(a={ }_{0} a . \equiv . a \varepsilon a\right)$

Now the last axiom which is to be stated is concerned with singular propositions consisting of two general descriptive names:

$$
\begin{aligned}
{[\phi \psi](\operatorname{trm}\langle\phi\rangle \varepsilon \operatorname{trm}\langle\psi\rangle . \equiv} & .[E x]\left(x={ }_{0} x \cdot \phi(x)\right) . \\
& {[x y]\left(x={ }_{0} x \cdot y={ }_{0} y \cdot \phi(x) \cdot \phi(y) \cdot \supset \cdot x={ }_{0} y\right) . } \\
& {\left.[x]\left(x={ }_{0} x \cdot \phi(x) \cdot \supset \cdot \phi(x)\right)\right)^{25} }
\end{aligned}
$$

As is easy to see, this reflects the very idea of Russellian theory of description, though it differs in form from the original definition we find in Principia Mathematica ${ }^{26}$. We should notice that we are in a position to state this axiom because we

24. For the mechanical method to decide, using the notion of semantic category, if a given series of symbols is a well-formed expression, cf. e.g. Ajdukiewicz [1935], Lambek [1958].

25. Notice again Lejewski's suggestion which claims that the theory of description is concerned not with the definite article 'the' but rather with the logical property of 'is'. This view is reflected here. Cf. Note 9.

26. The definition we find in Principia Mathematica is : 
have at hand 'individual identity'.

T1 $[a b](a \varepsilon b . \equiv . a \varepsilon \operatorname{trm}\langle\varepsilon f b+\rangle)$

T2 $[a b](a \varepsilon b . \equiv . \operatorname{trm}\langle\varepsilon+a \dagger\rangle \varepsilon b)$

T3 $[a b](a \varepsilon \operatorname{trm}\langle\varepsilon(b)\rangle . \equiv . \operatorname{trm}\langle\varepsilon(a)\rangle \varepsilon \operatorname{trm}\langle\varepsilon(b)\rangle)$

T4 $[a b](a \varepsilon b . \equiv \operatorname{trm}\langle\varepsilon f a \dagger\rangle \varepsilon \operatorname{trm}\langle\varepsilon+b \nmid\rangle)$

T5 $[a b](a \varepsilon b . \supset . a \varepsilon a)$

Pr. $\quad 1 . \quad a \varepsilon b$

2. $\operatorname{trm}\langle\varepsilon+a \nmid\rangle \varepsilon \operatorname{trm}\langle\varepsilon+b+\rangle$

3. $[E x]\left(x={ }_{0} x . \varepsilon f a \dagger(x)\right)$.

$[2, \mathrm{~A} 6]$

4. $[x y]\left(x={ }_{0} x \cdot y={ }_{0} y \cdot \varepsilon f a \nmid(x) \cdot \varepsilon f a \nmid(y) \cdot \supset \cdot x={ }_{0} y\right)$

[2, A6]

5. $[x]\left(x={ }_{0} x . \varepsilon f a\right)(x)$..$\left.\left.\varepsilon f a\right)(x)\right)$

[Thesis]

6. $\operatorname{trm}\langle\varepsilon(a)\rangle \varepsilon \operatorname{trm}\langle\varepsilon f a)\rangle$

$[3,4,5, \mathrm{~A} 6]$

7. $a \varepsilon a$

$[\mathrm{T} 4,5]$

T6 $[a b]\left(a \varepsilon b . \supset . a={ }_{0} a\right)$

T7 $[a b](\varepsilon+b+(a) . \supset . a \varepsilon a)$

T8 $[a b](\varepsilon+b+(a) . \equiv . a \varepsilon a . \varepsilon+b+(a))$

T9 $[a b]\left(\varepsilon+b+(a) . \equiv . a={ }_{0} a . \varepsilon+b+(a)\right)$

$\mathrm{T} 10 \quad[a b c](a \varepsilon b . b \varepsilon c . \supset . a \varepsilon c)$

1. $a \varepsilon b$

2. $b \varepsilon c$

3. $\operatorname{trm}\langle\varepsilon+a+\rangle \varepsilon \operatorname{trm}\langle\varepsilon+b+\rangle$

[1, T4]

4. $\operatorname{trm}\langle\varepsilon+b+\rangle \varepsilon \operatorname{trm}\langle\varepsilon+c+\rangle$

5. $[E x]\left(x={ }_{0} x . \varepsilon f a+(x)\right)$

$[3, \mathrm{A6}]$

6. $[x y]\left(x={ }_{0} x \cdot y={ }_{0} y \cdot \varepsilon f a \nmid(x) \cdot \varepsilon f a \dashv(y) \cdot \supset \cdot x={ }_{0} y\right)$

7. $[x]\left(x={ }_{0} x . \varepsilon f a+(x)\right.$. ว. $\left.\varepsilon(b)(x)\right)$

8. $[x]\left(x={ }_{0} x . \varepsilon+b\right)(x)$..$\left.\varepsilon(c)(x)\right)$

9. $[x]\left(x={ }_{0} x . \varepsilon f a \nmid(x)\right.$..$\left.\varepsilon f c \nmid(x)\right)$

10. $\operatorname{trm}\langle\varepsilon f a+\rangle \varepsilon \operatorname{trm}\langle\varepsilon f c+\rangle$

11. $a \varepsilon c$

T11 $[E a](\phi(a)) \cdot[x](\phi(x) \cdot \supset \cdot \phi(x)) \cdot[E x](\phi(x)) \cdot[x y]\left(\phi(x) \cdot \phi(y) \cdot \supset \cdot x={ }_{0} y\right) . \supset$. $[x](\phi(x) \supset \phi(x))$

Pr. 1. $\quad[E a](\phi(a))$

2. $[x](\phi(x) . \supset . \psi(x))$ 
3. $[E x](\varphi(x))$

4. $[x y]\left(\psi(x) \cdot \psi(y) \cdot \supset \cdot x={ }_{0} y\right)$

5. $\phi(x)$ [sup.]

6. $\sim \phi(x)$

7. $\phi(a)$

[a. sup.]

8. $\psi(a)$

9. $a={ }_{0} x$

$[2 ; x / \mathrm{a}, 7]$

10. $\sim \phi(a)$

$[4 ; x / a, y / x, 8,5]$

11. contradiction

$[9, \mathrm{~A} 2,6]$

$[7,10]$

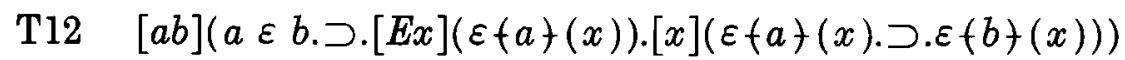

[T4, A6, T9]

$\mathrm{T} 13[b c]\left(b \varepsilon c . \supset .[E x](\varepsilon+b+(x)) \cdot[x y]\left(\varepsilon+b+(x) \cdot \varepsilon+b+(y) . \supset . x={ }_{0} y\right)\right)[\mathrm{T} 4, \mathrm{A6}, \mathrm{T} 9]$

$\mathrm{T} 14 \quad[a b](a \varepsilon b . b \varepsilon$ c. $\supset .[x](\varepsilon+b+(x) . \supset . \varepsilon+a \dagger(x)))$

$[\mathrm{T} 11 ; \phi / \varepsilon f a f, \phi / \varepsilon+b \nmid, \mathrm{T} 12, \mathrm{~T} 13]$

$\mathrm{T} 15[a b c](a \varepsilon b . b \varepsilon c . \supset . b \varepsilon a)$

[T13, T14, T9, A6, T4]

T16 $[a b c](a \varepsilon b . b \varepsilon b . \supset . b \varepsilon a)$

T17 $[a b c]\left(a \varepsilon b . b={ }_{0} b . \supset . b \varepsilon a\right)$

[T16, A5]

T18 $[a b]\left(a={ }_{0} b . \equiv . a \varepsilon b . b \varepsilon a\right)$

Pr. 1.1. $a={ }_{0} b$

[sup.]

1.2. $a={ }_{0} a$

$[1,1, \mathrm{~T} 13]$

1.3. $a \varepsilon a$

$[1,2, \mathrm{~A} 5]$

1.4. $\varepsilon f a f(a)$

$[1,3, \mathrm{A4}]$

1.5. $\varepsilon f a f(a) . \equiv \varepsilon f a f(b)$

$[1.1, \mathrm{~A} 2 ; \phi / \varepsilon(\mathrm{a})]$

1.6. $\varepsilon f a+(b)$

$[1.5,1.4]$

1.7. $b \varepsilon a$

$[1.6, \mathrm{~A} 4]$

1.8. $a \varepsilon b$

[like 1.1-1.7]

1.9. $a \varepsilon b . b \varepsilon a$

1. $a={ }_{0} b . \supset . a \varepsilon b . b \varepsilon a$

[1.1-1.9]

2.1. $a \varepsilon b . b \varepsilon a$

[sup.]

2.2. $[x y]\left(\varepsilon+a+(x) \cdot \varepsilon+a+(y) \cdot \supset . x={ }_{0} y\right)$

[2.1, T4, A6, T9]

2.3. $[x]\left(\varepsilon f a+(x) \cdot \varepsilon f a \dashv(a) \cdot \supset . x={ }_{0} a\right)$

$[2.2 ; y / a]$

2.4. $a \varepsilon a$

$[2.1, \mathrm{~T} 5]$

2.5. $\varepsilon f a+(a)$

$[2.4, \mathrm{~A} 4]$

2.6. $[x]\left(\varepsilon+a+(x) . \supset . x={ }_{0} a\right)$

$[2.3,2.5]$

2.7. $\varepsilon f a+(b) \cdot \supset . b={ }_{0} a$

$[2.6 ; x / b]$

2.8. $\varepsilon f a+(b)$

$[2.1, \mathrm{~A} 4]$

2.9. $b={ }_{0} a$

$[2.7,2.8]$

2.10. $a={ }_{0} b$

$[2.9, \mathrm{TI} 1]$ 
2. $a \varepsilon b . b \varepsilon a . \supset . a={ }_{0} b$

3. $a={ }_{0} b . \equiv . a \varepsilon b . b \varepsilon a$

T19 $[a b c]\left(a \varepsilon b \cdot b={ }_{0} b \cdot \supset \cdot b={ }_{0} a\right)$

T20 $[a b]\left(a={ }_{0} a . \equiv .[E x](\varepsilon+a+(x))\right.$.

$$
\left.\left.[x y](\varepsilon+a)(x) \cdot \varepsilon+a+(y) \cdot \supset \cdot x={ }_{0} y\right)\right)
$$

[A5, T4, A6, T9]

T21 $\left.[a \phi]\left(a={ }_{0} a \cdot[x](\varepsilon+a+(x) \cdot \supset \cdot \phi(x))\right) . \equiv . a={ }_{0} a \cdot \phi(a)\right)$

Pr. 1.1. $a={ }_{0} a$

1.2. $[x](\varepsilon f a+(x) . \supset . \phi(x))$

[sup.]

1.3. $a \in a$

1.4. $\varepsilon f a+(a)$

$[1.3, \mathrm{~A} 4]$

1.5. $\phi(a)$

1. $a \dot{=}_{0} a \cdot[x](\varepsilon f a \dashv(x) . \supset \cdot \phi(x)) \cdot \supset \cdot a={ }_{0} a \cdot \phi(a)$

$[1.1,1.2-1.5]$

2.1. $\quad a={ }_{0} a . \phi(a)$

[sup.]

2.2. $\varepsilon f a+(x)$

[sup.]

2.3. $x \in a$

2.4. $x={ }_{0} a$

$[\mathrm{T} 19 ; a / x, b / a, 2.3,2.1]$

2.5. $\phi(x)$

$[2.4, \mathrm{~A} 2,2.1]$

2. $a={ }_{0} a \cdot \phi(a) \cdot \supset \cdot[x](\varepsilon+a \nmid(x) \cdot \supset \cdot \phi(x))$

3. $\quad a={ }_{0} a \cdot \phi(a) \cdot \supset \cdot[x](\varepsilon f a \nmid(x) \cdot \supset \cdot \phi(x)) \cdot a={ }_{0} a$

4. $\left.a={ }_{0} a \cdot[x](\varepsilon f a \nmid(x) . \supset \cdot \phi(x))\right) . \equiv . a={ }_{0} a \cdot \phi(a)$

T22 $[a \phi]\left(a \varepsilon \operatorname{trm}\langle\phi\rangle . \equiv . a={ }_{0} a \cdot \phi(a)\right)$

Pr. 1. $a \varepsilon \operatorname{trm}\langle\phi\rangle . \equiv \operatorname{trm}\langle\varepsilon f a \dagger\rangle \varepsilon \operatorname{trm}\langle\phi\rangle$

2. $\quad \equiv .[E x](\varepsilon+a+(x))$.

$$
\begin{aligned}
& {[x y]\left(\varepsilon+a \dashv(x) \cdot \varepsilon f a \dashv(y) \cdot \supset \cdot x={ }_{0} y\right)} \\
& {[x](\varepsilon+a \dashv(x) \cdot \supset \cdot \phi(x))}
\end{aligned}
$$

3.

$$
\equiv . a={ }_{0} a \text {. }
$$

$$
[x](\varepsilon f a)(x) . \supset . \phi(x))
$$

4.

$$
\equiv . a={ }_{0} a . \phi(a)
$$

$\mathrm{T} 23[a \phi](a \varepsilon \operatorname{trm}\langle\phi\rangle . \equiv . a \varepsilon a . \phi(a))$

[T22, A5]

T24 $[a b](\operatorname{trm}\langle\varepsilon f a \nmid\rangle \varepsilon \operatorname{trm}\langle\varepsilon+b \nmid\rangle . \equiv .[E x](\varepsilon f a \nmid(x))$.

$$
\begin{aligned}
& {[x y]\left(\varepsilon+a+(x) \cdot \varepsilon f a f(y) \cdot \supset \cdot x={ }_{0} y\right) .} \\
& [x](\varepsilon f a+(x) \cdot \supset \cdot \varepsilon+b+(x)))
\end{aligned}
$$

T25 $[a b](\operatorname{trm}\langle\varepsilon f a \nmid\rangle \varepsilon \operatorname{trm}\langle\varepsilon f b \dashv\rangle . \equiv .[E x](x \varepsilon a)$.

$$
[x y]\left(x \in a . y \in \text { \& } a . \supset . x={ }_{0} y\right)
$$

27. Prof. R. E. Clay pointed out in a personal communication that this proof can be shortened if we state $a={ }_{0} b . \equiv . b={ }_{0} a$ separately which is an immediate corollary of T8. 


$$
[x](x \in a . \supset . x \varepsilon b))
$$

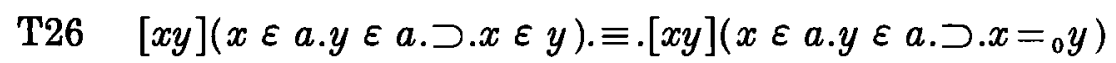

T27 $[a b](\operatorname{trm}\langle\varepsilon f a)\rangle \varepsilon \operatorname{trm}\langle\varepsilon f b+\rangle . \equiv .[E x](x \varepsilon a)$.

$$
\begin{aligned}
& {[x y](x \in a . y \varepsilon a . \supset . x \varepsilon y)} \\
& [x](x \varepsilon a . \supset . x \varepsilon b))
\end{aligned}
$$

T28 $[a b](a \varepsilon b . \equiv .[E x](x \varepsilon a)$.

$$
\begin{aligned}
& {[x y](x \varepsilon a . y \varepsilon a . \supset . x \varepsilon y)} \\
& [x](x \varepsilon a . \supset . x \varepsilon b))
\end{aligned}
$$

Thus we arrived at the axiom of Leśniewski's Ontology.

Formally stated, what we have achieved so far can be summarized in the following theorem :

Theorem 1: If we widen the range of names from individual names to names in general, then predicate calculus with non-reflexive identity enriched as indicated implies the axiom of Leśniewski's Ontology.

Thus Ontology finds its place in a natural extension of LI with description.

Now we define the notion of extensional identity in the following way:

DI $[a b](a=b . \equiv .(x)(x \varepsilon a . \equiv . x \varepsilon b))$

It is easy to check that :

TI1 $[a b](a=a)$

TI2 $[a b](a=b . \supset . b=a)$

TI3 $[a b c](a=b . b=c . \supset . a=c)$

Thus $\mathrm{LI}^{+}$is a system with total reflexive identity.

To the axiom AI3 corresponds the following axiom of extensionality :

$\mathrm{AE} \quad[a b](a=b . \supset \cdot[\phi](\phi(a) . \equiv . \phi(b))$

Even though this axiom looks evident and universally acceptable, it will appear that it need not be so. AE seems to be too extensional. And it will be shown that we can think of at least three different systems of Ontology (and two exensions of LI) due to the logical power of AE.

To finish this chapter I will mention some properties of individual identity.

T29 $[x y]\left(x={ }_{0} y \cdot \supset . x \varepsilon x \cdot[\phi](\phi(x) . \equiv . \phi(y))\right.$

T30 $[x y]\left(x \in x \cdot[\phi](\phi(x) . \equiv . \phi(y)) . \supset \cdot x={ }_{0} y\right)$

Pr. $\quad 1 . \quad x \in x \cdot[\phi](\phi(x) \equiv . \phi(y))$

2. $\varepsilon+x+(x)$

3. $\varepsilon f x+(x) . \equiv . \varepsilon+x+(y)$ 
6. $x={ }_{0} y$

T31 $[x y]\left(x={ }_{0} y . \equiv . x \in x \cdot[\phi](\phi(x) . \equiv . \phi(y))\right)$

[T29, T30]

\section{Comparing $\mathrm{LI}^{+}$with Ontology}

We now compare $\mathrm{LI}^{+}$with Ontology. To do this, we will consider three systems of Ontology.

5.1. We reconfirm that the axioms of $\mathrm{LI}^{+}$are the following:

$\mathrm{A} 1[a b]\left(a={ }_{0} b . \equiv .[E x]\left(\dot{x}={ }_{0} a\right)\right.$.

$$
\begin{aligned}
& {[x y]\left(x={ }_{0} b \cdot y={ }_{0} b \cdot \supset \cdot x={ }_{0} y\right) .} \\
& \left.[x]\left(x={ }_{0} a \cdot \supset \cdot x={ }_{0} b\right)\right)
\end{aligned}
$$

A2 $[x y]\left(x={ }_{0} y \cdot \supset \cdot[\phi](\phi(x) . \equiv . \phi(y))\right)$

A3 $[\phi a](\phi(a) . \equiv . \phi(\operatorname{trm}\langle\varepsilon+a)\rangle))$

$\mathrm{A} 4 \quad[x a](x \in a . \equiv . \varepsilon(a)(x))$

A5 $[a]\left(a={ }_{0} a . \equiv . a \varepsilon a\right)$

A6 $[\phi \psi]\left(\operatorname{trm}\langle\phi\rangle \varepsilon \operatorname{trm}\langle\phi\rangle . \equiv .[E x]\left(x={ }_{0} x . \phi(x)\right)\right.$.

$$
\begin{aligned}
& {[x y]\left(x={ }_{0} x \cdot y={ }_{0} y \cdot \phi(x) \cdot \phi(y) \cdot \supset \cdot x={ }_{0} y\right)} \\
& {[x]\left(x={ }_{0} x \cdot \phi(x) \cdot \supset \cdot \phi(x)\right)^{28}}
\end{aligned}
$$

5.2. Now let us refer to Ontology as OL the sole axiom of which is :

A0 $[a b](a \varepsilon b . \equiv .[E x](x \in a)$.

$$
\begin{aligned}
& {[x y](x \in a . y \varepsilon \quad a . \supset . x \in y)} \\
& [x](x \in a . \supset . x \in b))
\end{aligned}
$$

with the following definitions:

D1 $[a b]\left(a={ }_{0} b . \equiv . a \varepsilon b . b \varepsilon a\right)$

D2 $[x a](\varepsilon f a \dashv(x) . \equiv x \varepsilon a)$

D3 $[a \phi](a \varepsilon \operatorname{trm}\langle\phi\rangle . \equiv . a \varepsilon a . \phi(a))^{29}$

Thus OL $=\langle\mathrm{AO}, \mathrm{D} 1, \mathrm{D} 2, \mathrm{D} 3\rangle$.

28. Notice that $\mathbf{A 1}$ is equivalent to ;

$\mathrm{Al} \#[a b]\left(a={ }_{0} b . \equiv .[E x] \cdot x={ }_{0} a \cdot x={ }_{0} b\right)$

or the conjunction of the following two theses;

$\mathrm{AIl}[a b]\left(a={ }_{0} b . \supset . b={ }_{0} a\right)$

$\mathrm{AI2}[a b c]\left(a={ }_{0} b . b={ }_{0} c \cdot \supset \cdot a={ }_{0} c\right)$

29. This definition is called ontological definition. On ontoloical definition, cf. Lejewski [1958], [1967], Shupecki [1955]. Strictly speaking, we have to require of $\mathrm{LI}^{+}$this style of definition, but we do not touch this point here, assuming that this is allowed 
5.3. Let us refer to Ontology the axioms of which are AO and the axiom of extensionality AE with DI, D1, D2, D3 as $\mathrm{OL}^{+}$.

DI $\quad[a b](a=b . \equiv .(x)(x \in a . \equiv . x \varepsilon b))$

$\mathrm{AE} \quad[a b](a=b . \supset \cdot[\phi](\phi(a) . \equiv . \phi(b))$

Thus $\mathrm{OL}^{+}=\langle\mathrm{AO}, \mathrm{DI}, \mathrm{AE}, \mathrm{D} 1, \mathrm{D} 2, \mathrm{D} 3\rangle$.

5.4. Then let us consider a restricted system of Ontology the axioms of which are $\mathrm{AO}$ and the following axiom of weak extensionality:

$\mathrm{AWE} \quad[\phi \mathrm{a}](\phi(\mathrm{a}) . \equiv . \phi(\operatorname{trm}\langle\varepsilon f a \dashv\rangle))^{30}$

and refer to it as $\mathrm{OLW}^{+}$. Further we assume that the definitions D1, D2, D3 are added to $\mathrm{OLW}^{+}$. Thus $\mathrm{OLW}^{+}=\langle\mathrm{AO}, \mathrm{AWE}, \mathrm{D} 1, \mathrm{D} 2, \mathrm{D} 3\rangle$.

Thus $\mathrm{OL}^{+}$is the strongest and $\mathrm{OL}$ is the weakest. $\mathrm{OLW}^{+}$finds its place in between.

Now we have the proposition:

Proposition 1: A1, A2, A4, A5, A6 of $\mathrm{LI}^{+}$are theses of $\mathrm{OL}$.

The following series of theses (TT1-TT12) is a proof of the proposition.

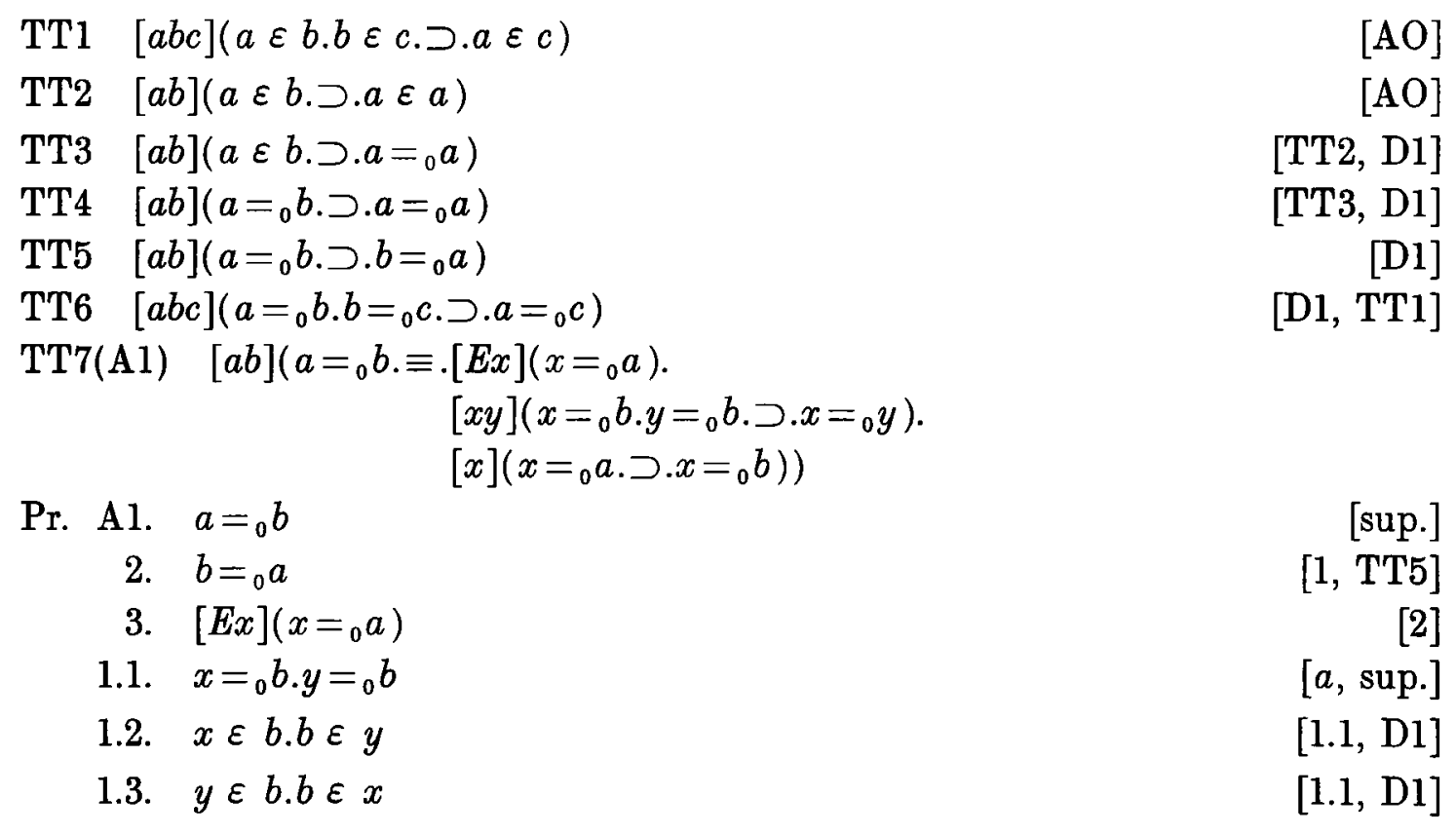

in LI. If we want to state this not as a rule of definition but as a formula, then the following will do:

$\mathrm{AD}[\phi]([E a]([x](x \varepsilon a . \equiv . x \varepsilon x . \phi(x))$

Thus stated, ontological definition is akin to set-theoretical operation of abstraction.

30. This is indeeed a restricted form of $\mathrm{AE}$. This follows from $\mathrm{AE}$ and $\mathrm{T} 1$. 
1.4. $x \varepsilon y . y \varepsilon x$

$[1.2,1.3, \mathrm{TT} 1]$

1.5. $x={ }_{0} y$

4. $x={ }_{0} b . y={ }_{0} b . \supset . x={ }_{0} y$

5. $[x y]\left(x={ }_{0} b \cdot y={ }_{0} b . \supset \cdot x={ }_{0} y\right)$

2.1. $x={ }_{0} a$

2.2. $x={ }_{0} b$

$[2.1,1, \mathrm{TT} 6]$

6. $x={ }_{0} a \cdot \supset \cdot x={ }_{0} b$

7. $[x]\left(x={ }_{0} a \cdot \supset . x={ }_{0} b\right)$

8. $[E x]\left(x={ }_{0} a\right)$.

$[x y]\left(x={ }_{0} b \cdot y={ }_{0} b . \supset \cdot x={ }_{0} y\right)$.

$[x]\left(x={ }_{0} a \cdot \supset \cdot x={ }_{0} b\right)$

Pr. B1. $[E x]\left(x={ }_{0} a\right)$.

$[3,5,7]$

2. $[x y]\left(x={ }_{0} b \cdot y={ }_{0} b . \supset \cdot x={ }_{0} y\right)$.

3. $[x]\left(x={ }_{0} a \cdot \supset \cdot x={ }_{0} b\right)$

4. $a={ }_{0} a$

[sup.]

5. $a={ }_{0} a \cdot \supset \cdot a={ }_{0} b$

[1, TT5, TT4]

6. $a={ }_{0} b$

$[3 ; x / a]$

TT8 $[x y]\left(x={ }_{0} y \cdot \supset \cdot[\phi](\phi(x) . \supset . \phi(y))\right)$

Pr. 1. $x={ }_{0} y$

[sup.]

2. $\phi(x)$

[sup.]

3. $x={ }_{0} x$

[1, TT4]

4. $x \varepsilon x$

$[3, \mathrm{D} 1]$

5. $x \varepsilon \operatorname{trm}\langle\phi\rangle$

$[2,4, \mathrm{D} 3]$

6. $y \varepsilon x$

$[1, \mathrm{D} 1]$

7. $y \varepsilon \operatorname{trm}\langle\phi\rangle$

$[5,6, \mathrm{TT} 1]$

8. $\phi(y)$

TT9(A2) $[x y]\left(x={ }_{0} y \cdot \supset \cdot[\phi](\phi(x) . \equiv . \phi(y))\right)$

TT10(A4) $[x a](x \varepsilon a . \equiv . \varepsilon f a+(x))$

$\mathrm{TT} 11(\mathrm{~A} 5) \quad[a]\left(a={ }_{0} a . \equiv . a \varepsilon a\right)$

$\mathrm{TT} 12(\mathrm{~A} 6) \quad[\phi \psi]\left(\operatorname{trm}\langle\phi\rangle \varepsilon \operatorname{trm}\langle\psi\rangle . \equiv .[E x]\left(x={ }_{0} x . \phi(x)\right)\right.$.

$$
\begin{aligned}
& {[x y]\left(x={ }_{0} x \cdot y={ }_{0} y \cdot \phi(x) \cdot \phi(y) \cdot \supset \cdot x={ }_{0} y\right)} \\
& \left.[x]\left(x={ }_{0} x \cdot \phi(x) \cdot \supset \cdot \phi(x)\right)\right)
\end{aligned}
$$

Pr. 1. $\operatorname{trm}\langle\phi\rangle \varepsilon \operatorname{trm}\langle\phi\rangle . \equiv .[E x](x \varepsilon \operatorname{trm}\langle\phi\rangle)$.

$[x y](x \varepsilon \operatorname{trm}\langle\phi\rangle . y \varepsilon \operatorname{trm}\langle\phi\rangle . \supset . x \varepsilon y)$.

$$
[x](x \varepsilon \operatorname{trm}\langle\phi\rangle . \supset . x \varepsilon \operatorname{trm}\langle\psi\rangle)
$$

$2 . \quad . \equiv .[E x](x \in x \cdot \phi(x))$.

$[x y](x \varepsilon x . \phi(x) . y \varepsilon y . \phi(y) . \supset . x \varepsilon y)$

$[x](x \in x . \phi(x) . \supset . x \varepsilon x . \phi(x))$ 
3.

$$
\begin{aligned}
. \equiv & {[E x](x \varepsilon x . \phi(x)) . } \\
& {[x y](x \varepsilon x . \phi(x) . y \varepsilon y . \phi(y) . \supset . x \varepsilon y . y \varepsilon x) } \\
& {[x](x \varepsilon x . \phi(x) . \supset . x \varepsilon x . \phi(x)) }
\end{aligned}
$$

4.

$$
\begin{aligned}
. \equiv & {[E x]\left(x={ }_{0} x \cdot \phi(x)\right) . } \\
& {[x y]\left(x={ }_{0} x \cdot \phi(x) \cdot y={ }_{0} y \cdot \phi(y) \cdot \supset \cdot x={ }_{0} y\right) } \\
& {[x]\left(x={ }_{0} x \cdot \phi(x) \cdot \supset \cdot x={ }_{0} x \cdot \psi(x)\right) }
\end{aligned}
$$

5.

$$
\begin{aligned}
. \equiv & {[E x]\left(x={ }_{0} x \cdot \phi(x)\right) . } \\
& {[x y]\left(x={ }_{0} x \cdot \phi(x) \cdot y={ }_{0} y \cdot \phi(y) \cdot \supset \cdot x={ }_{0} y\right) } \\
& {[x]\left(x={ }_{0} x \cdot \phi(x) \cdot \supset \cdot \phi(x)\right) }
\end{aligned}
$$

From Theorem 1 of the preceding chapter we have the following proposition :

Proposition 2: AO follows from A1, A2, A3, A4, A5, A6.

Notice that $\mathrm{A} 3=\mathrm{AWE}$, and recall that $\mathrm{D} 1, \mathrm{D} 2, \mathrm{D} 3$ are all theses of $\mathrm{LI}^{+}$.

From Propositions 1 and 2 we get:

Theorem $2: \mathrm{LI}^{+}$is inferentially equivalent with $\mathrm{OLW}^{+}$.

OL is too weak in that we cannot treat ' $a$ ' and ' $\operatorname{trm}\langle\varepsilon f a \dagger\rangle$ as coextensional. $\mathrm{OL}^{+}$is in this context unnecessarily strong. It happens at times that we wish to distinguish 'unicorn' from 'round square'. $\mathrm{LI}^{+}$, which is a natural extension of LI, is Ontology with the axiom of weak extensionality AWE.

\section{Simplified $\mathrm{LI}^{+}$as an extremely extensional system}

Let us recall that the following are theses in $\mathrm{LI}^{+}$:

TTT1(T4) $[a b](a \varepsilon b . \supset . a \varepsilon a)$

TTT2(T7) $[a b c](a \varepsilon b . b \varepsilon c . \supset . a \varepsilon c)$

From these we obtain :

TTT3(AO\#) $[a b](a \varepsilon b . \equiv .[E c](a \varepsilon c . c \varepsilon b))$

Pr. 1.1. $a \varepsilon b$

[sup.]

1.2. $a \varepsilon a$

1.3. $a \varepsilon a . a \varepsilon b$

1.4. $[E c](a \varepsilon c . c \varepsilon a)$

1. $a \varepsilon b . \supset .[E c](a \varepsilon c . c \varepsilon b)$

2.1. $[E c](a \varepsilon c . c \varepsilon b)$

2.2. $a \varepsilon b$ 
2. $[E c](a \varepsilon c . c \varepsilon b) . \supset . a \varepsilon b$

3. $a \varepsilon b . \equiv .[E c](a \varepsilon c . c \varepsilon b)$

Notice that $\mathrm{AO \#}$ functions in the presence of $\mathrm{AE}$ as a single axiom of Ontology ${ }^{31}$.

Let us notice that all the axioms made use of in showing $\mathrm{T} 4$ and $\mathrm{T} 7$ were A3 and A6.

Let us consider a set of the following theses :

TTT4(A3) $[\phi a](\phi(a) . \equiv . \phi(\operatorname{trm}\langle\varepsilon+a \nmid\rangle))$

$\operatorname{TTT5}(\mathrm{A} 6) \quad[\phi \psi]\left(\operatorname{trm}\langle\phi\rangle \varepsilon \operatorname{trm}\langle\psi\rangle . \equiv .[\mathrm{E} x]\left(x={ }_{0} x . \phi(x)\right)\right.$.

$$
\begin{aligned}
& {[x y]\left(x={ }_{0} x \cdot y={ }_{0} y \cdot \phi(x) \cdot \phi(y) \cdot \supset \cdot x={ }_{0} y\right) .} \\
& \left.[x]\left(x={ }_{0} x \cdot \phi(x) \cdot \supset \cdot \phi(x)\right)\right)
\end{aligned}
$$

TTT6(DI) $[a b](a=b . \equiv .(x)(x \varepsilon a . \equiv . x \varepsilon b))$

TTT7(AE) $[a b](a=b . \supset .[\phi](\phi(a) . \equiv . \phi(b)))$

Notice that TTT4 follows from TTT7 if we pose the following:

TTT4\#(A3\#) $[a](a=\operatorname{trm}\langle\varepsilon f a \dagger\rangle)$

and TTT4\# follows from TTT4, so that in the presence of TTT6 and TTT7, TTT4 is equivalent to TTT4\#.

Now let us consider a system the axioms of which are <TTT4, TTT5, TTT6, TTT7〉, i.e. 〈A3, A6, DI, AE〉,

and refer to it as LIE.

Proposition 3 : LIE has AO as its thesis.

Proof: TTT3 is a thesis of LIE, for TTT1 and TTT2 are theses of LIE. LIE has $\mathrm{AE}$ as a thesis. AO follows form TTT3 and AE. Hence AO is a thesis of LIE.

It is easy to show that $\mathrm{A} 3$ is a thesis of $\mathrm{OL}^{+}$. Thus we obtain the following proposition :

Proposition 4: Every thesis of LIE is a thesis of $\mathrm{OL}^{+}$.

Now we $\operatorname{add}^{32}$ to LIE the following definitions :

D1 $[a b] . a={ }_{0} b . \equiv . a \varepsilon b . b \varepsilon a$

D2 $[x a](\varepsilon+a)(x) . \equiv x \varepsilon a)$

D3 $[a \phi](a \varepsilon \operatorname{trm}\langle\phi\rangle . \equiv . a \varepsilon a . \phi(a))$

31. Cf. esp. Sobociński [1934].

32. To speak more exactly, we have to state the rule of definitions in terms of ' $\varepsilon$ '. Cf. Note 29. 
So we consider the system 〈TTT4, TTT5, TTT6, TTT7, D1, D2, D3〉 (i.e. 〈A3, A6, $\mathrm{DI}, \mathrm{AE}, \mathrm{D} 1, \mathrm{D} 2, \mathrm{D} 3\rangle)$ and we refer to it as $\mathrm{LIE}^{+}$. Obviously,

Proposition 5 : Every thesis of $\mathrm{OL}^{+}$is a thesis of $\mathrm{LIE}^{+}$.

Proposition 6: Every thesis of $\mathrm{LIE}^{+}$is a thesis of $\mathrm{OL}^{+}$.

Proof: From Proposition 1 we have that TTT5(A6) is a thesis of OL, so that TTT5(A6) and TTT7(AE) with TTT6(DI) are theses of $\mathrm{OL}^{+}$. D1, D2, D3 are common to both $\mathrm{LIE}^{+}$and $\mathrm{OL}^{+}$. TTT4(A3) is a thesis of $\mathrm{OL}^{+}$. Its proof is as following :

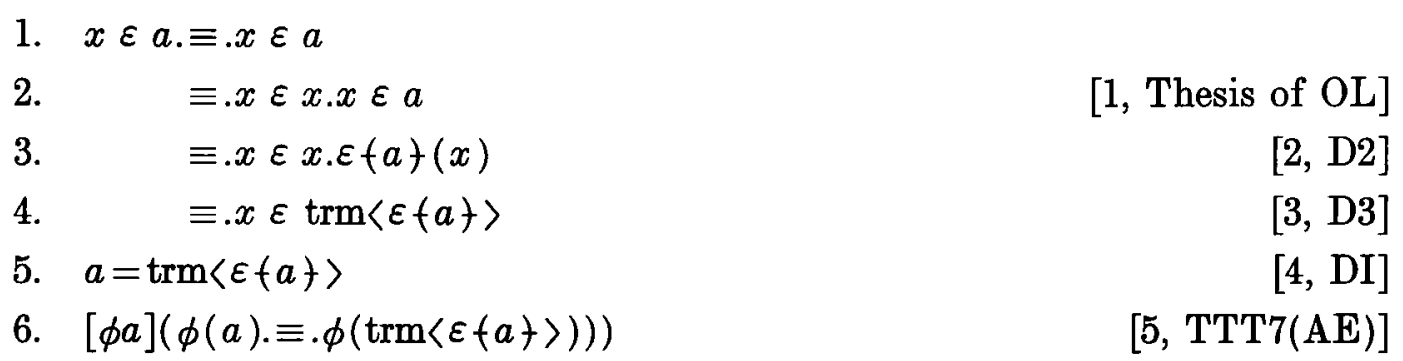

Hence we arrived to the following theorem:

Theorem 3: $\mathrm{LIE}^{+}$is inferentially equivalent to $\mathrm{OL}^{+}$.

From this we have that if LI is supposed to be extremely extensional, i.e. it fulfills $\mathrm{AE}$ with DI, then A3 and A6 (with D1, D2, D3) suffice as an extension of LI in which we have verb/name-name/verb conversion with singular proposition formative ' $\varepsilon$ '.

One more thing should be mentioned with respect to this point to finish this paper. Compared to $\mathrm{LIE}^{+}, \mathrm{LI}^{+}$, in my opinion, is not extremely extensional. Indeed what is reuired in $\mathrm{LI}^{+}$is only that a context is indifferent to changing ' $a$ ' to 'trm $\langle\varepsilon f a t\rangle$ ' and not that a context remains unaffected by any change of terms extensionally identical, while this is just what $\mathrm{LIE}^{+}$requires. Thus if one does not mind being an extreme extensionalist, he can extend LI in a natural way with <A3, $\mathrm{A} 6, \mathrm{DI}, \mathrm{AE}>$ for him to be able to deal with general name and name/verb-verb/name conversion with singular proposition formative ' $\varepsilon$ '. But if one does not want to go so far, he may remain within $\mathrm{LI}^{+}$to do that job. $\mathrm{LIE}^{+}$is a system which an extreme extensionalist can choose as a natural extension of $\mathrm{LI}$, while $\mathrm{LI}^{+}$is a natural extension which can be accepted by a moderate extensionalist. 


\section{References}

Ajdukiewicz, K. [1935] : Die Syntaktische Konnexität, Studia Philosophica, Vol. 1, 1-28 Gochet, P. [1980]: Outline of a Nominalist Theory of Propositions, Reidel Henry, D.P. [1972] : Medieval Logic and Metaphysics, Hutchinson University Library Hintikka, J. [1969] : Existential Presupposition And Their Elimination, in Models For Modalities, 23-44, Reidel

[1983]: "Is", Semantical Games, and Semantical Relativity, in The Game of Language, Hintikka, J. and Kulas, J. (eds.), 161-200, Reidel, 1983

Hiż, H. [1977] : Description in Russell's Theory and in Ontology, Studia Logica, 36, 271-283

Kaplan, D. [1970] : What Is Russell's Theory of Description?, in Physics, Logic and History, Yourgrau and Breck (eds.), N.Y. 227-295

Lambek, J. [1958] : The Mathematics of Sentence Structure, American Mathematical Month$l y, 65,154-170$

Lejewski, C. [1958] : On Leśniewski's Ontology, Ratio, Vol. 1, No. 2, 150-176. 1958 $35,14-29$ [1960] : A Re-Examination of The Russellian Theory of Descriptions, Philosophy, [1967] : A Theory of Non-Reflexive Identity And Its Ontological Ramifications, Grundfragen der Wisseschaften und ihre Wurzeln in der Metaphysik, 65-102

McCall, S. (ed.) [1967] : Polish Logic 1920-1939, Oxford

Quine, W.V.O. [1948]: On what there is, reprinted in From a Logical Point of View, 1953

[1968]: Existence and Quantification, reprinted in Ontological Relativity and Other Essays, 91-113, Columbia UP, 1979

Russell, B. (1905) : On Denoting, in Logic and Knowledge, 1956, Routlegde \& Keagan Paul, 41-46

[1919]: Introduction to Mathematical Philosophy,

Stupecki, J. [1955] : S. Leśniewski's Calculus of Names, Studia Logica, Vol. I, 44-111

Sobociński, B. [1934] : Successive Simplifications of the Axiom-System of Leśniewski's Ontology, Reprinted in Polish Logic, 188-200

Sommers, F. [1982]: The Logic of Natural Language, Clarendon Press

Waragai, T. [1985] : The Ontological Law Of Contradiction And Its Logical Structure, The Annals of the Japan Association for Philosophy of Science, Vol. 5, No. 5, 43-58

[1987]: Ontology Based on Non-Reflexive Identity and Product Name Functor, The Annals of the Japan Association for Philosophy of Science

Whitehead, Russell [1910] : Principia Mathematica, Vol. 1, Cambridge (Second edition, 1925) 